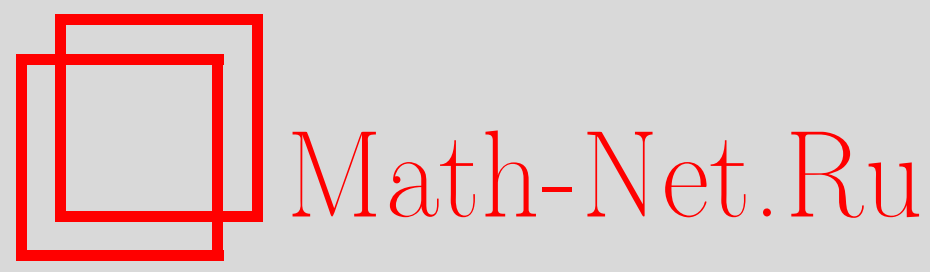

А. А. Туганбаев, Кольца, над которыми все циклические модули вполне целозамкнуты, Дискрет. матем., 2011, том 23, выпуск 3, 120-137

DOI: https://doi.org/10.4213/dm1156

Использование Общероссийского математического портала Math-Net.Ru подразумевает, что вы прочитали и согласны с пользовательским соглашением http://www.mathnet.ru/rus/agreement

Параметры загрузки:

IP: 54.92 .164 .108

26 апреля 2023 г., $13: 15: 33$ 


\title{
Кольца, над которыми все циклические модули вполне целозамкнуты
}

\author{
() 2011 г. А. А. Туганбаев
}

Описаны кольца, над которыми все циклические модули вполне целозамкнуты.

\section{1. Введение}

Все кольца предполагаются ассоциативными и с ненулевой единицей, а модули - унитарными. Выражения типа $A$ - цепное кольцо означают, что $A_{A}$ и ${ }_{A} A-$ цепные модули. Модуль $X$ называется инъективным относительно модуля $Y$ (или $Y$-инъективным), если для любого подмодуля $Y_{1}$ модуля $Y$ все гомоморфизмы $Y_{1} \rightarrow X$ продолжаются до гомоморфизмов $Y \rightarrow X$. Модуль $M$ над кольцом $A$ называется инъективным, если $M$ инъективен относительно любого $A$-модуля. Модуль называется самоинъективным (или квазиинъективным), если он инъективен относительно себя. В [6] описаны кольца, над которыми все циклические правые модули инъективны. В [5] описаны кольца, над которыми все циклические правые модули самоинъективны. Над кольцом вычетов $\mathbf{Z} / 4 \mathbf{Z}$ все циклические модули самоинъективны, но самоинъективный циклический $\mathbf{Z} / 4 \mathbf{Z}$-модуль $2 \mathbf{Z} / 4 \mathbf{Z}$ не инъективен.

Модуль $X$ называется вполне целозамкнутым, если для любого его подмодуля $Y$ каждый гомоморфизм $Y \rightarrow X$, переводящий в себя некоторый существенный подмодуль из $Y$, продолжается до гомоморфизма $X \rightarrow X$. Терминология объясняется тем, что коммутативная область $A$ с полем частных $Q$ является вполне целозамкнутым $A$-модулем в точности тогда, когда область $A$ - вполне целозамкнутое (в классическом смысле) подкольцо в поле $Q$ (см. лемму 14). Ясно, что каждый самоинъективный модуль вполне целозамкнут. Над кольцом целых чисел $\mathbf{Z}$ все циклические модули вполне целозамкнуты, но вполне целозамкнутый циклический $\mathbf{Z}$-модуль $\mathbf{Z}$ не самоинъективен.

Основным результатом данной работы является теорема 1, описывающая кольца, над которыми все циклические модули вполне целозамкнуты.

Теорема 1. Для кольиа А равносильнь следующие условия:

(1) все циклические правые А-модули и все цииклческие левые А-модули являются вполне целозамкнутыми модулями;

(2) $A=A_{1} \times \ldots \times A_{n}$, где либо $A_{i}-$ простое артиново кольцьо, либо $A_{i}-$ инвариантное иепное кольичо с не более чем двумя первичными идеалами, причем для любого нильпотентного идеала B кольца $A_{i}$ факторкольцо $A_{i} / B$ самоинъективно, либо $A_{i}$ - инвариантная вполне иелозамкнутая область, у которой для любого ненулевого 
идеала В факторкольцо $A_{i} / B$ является конечным прямым произведением инвариантных ияепных колец $A_{i 1}, \ldots, A_{i k(i)}$, причем кольцо $A_{i j}$ либо самоинъективно, либо является областью с не более чем двумя первичными идеалами, $i=1, \ldots, n$, $j=1, \ldots, k(i)$.

Например, все коммутативные дедекиндовы области и все артиновы кольца главных правых (левых) идеалов удовлетворяют теореме 1. В частности, кольцо целых чисел $\mathbf{Z}$ и все кольца вычетов $\mathbf{Z} / n \mathbf{Z}$ удовлетворяют теореме 1 .

Приведем необходимые определения и обозначения. Модуль $M$ называется цепным, если в $M$ любые два подмодуля сравнимы по включению. Модуль $M$ называется инвариантным, если в $M$ все подмодули вполне инвариантны. Ясно, что кольцо инвариантно справа (слева) в точности тогда, когда все его правые (левые) идеалы являются идеалами. В частности, все коммутативные кольца инвариантны. Модуль $M$ называется малоинъективным, если каждый эндоморфизм любого его подмодуля продолжается до эндоморфизма модуля $M$. Модуль $M$ называется малопроективным, если каждый эндоморфизм любого его фактормодуля поднимается до эндоморфизма модуля $M$; это означает, что для каждого эндоморфизма $\bar{f}$ произвольного фактормодуля $M / X$ модуля $M$ существует такой эндоморфизм $f$ модуля $M$, что $h f=\bar{f} h$, где $h: M \rightarrow M / X-$ естественный эпиморфизм.

Подмодуль $Y$ модуля $X$ называется существенным, если $Y$ имеет ненулевое пересечение с каждым ненулевым подмодулем в $X$. В этом случае говорят, что $M$ есть существенное расширение модуля $X$. Если инъективный модуль $M$ есть существенное расширение модуля $X$, то $M$ называется инъективной оболочкой модуля $X$. Хорошо известно, что каждый модуль имеет инъективную оболочку, причем она единственна с точностью до изоморфизма. Кроме того, каждый инъективный модуль, содержащий модуль $X$, также содержит некоторое свое прямое слагаемое, являющееся инъективной оболочкой модуля $X$. Модуль $M$ называется равномерным, если в $M$ любые два ненулевых подмодуля имеют ненулевое пересечение. Ясно, что модуль равномерен в точности тогда, когда все его ненулевые подмодули существенны. Кроме того, равномерные справа области совпадают с правыми областями Оре.

Модуль $M$ с инъективной оболочкой $E$ называется квазинепрерывным [4] (или $\pi$-инъективным [3]) при выполнении следующих эквивалентных условий (см. [4, 3]):

(1) для любого подмодуля $X$ модуля $M$ каждый идемпотентный эндоморфизм модуля $X$ продолжается до идемпотентного эндоморфизма модуля $M$;

(2) для любого подмодуля $X$ модуля $M$ каждое конечное прямое разложение $X=X_{1} \oplus \ldots \oplus X_{n}$ продолжается до прямого разложения $M=M_{1} \oplus \ldots \oplus M_{n}$;

(3) $f(M) \subseteq M$ для любого идемпотента кольца $\operatorname{End}(E)$;

(4) $M=\bigoplus_{i \in I}\left(M \cap E_{i}\right)$ для любого прямого разложения $E=\bigoplus_{i \in I} E_{i}$.

Для любого подмножества $X$ правого (соответственно левого) $A$-модуля $M$ через $r(X)$ (соответственно $l(X))$ обозначается правый (соответственно левый) аннулятор множества $X$ в $A$, т.е. $r(X)$ (соответственно $l(X))$ - правый (соответственно левый) идеал кольца $A$, состоящий из всех элементов $a \in A$, аннулирующих $X$.

Подкольцо $A$ кольца $Q$ называется классически вполне целозамкнутым справа в $Q$, если $A$ содержит каждый такой элемент $q \in Q$, что $q^{n} a \in A$ для некоторого неделителя нуля $a \in A$ и для всех $n \in \mathbf{N}$. Подкольцо $A$ кольца $Q$ называется вполне целозамкнутым 
справа в $Q$, если $A$ содержит каждый такой элемент $q \in Q$, что $q B \subseteq B$ для некоторого существенного правого идеала $B$ кольца $A$. Кольцо $A$ называется регулярным, если $a \in a A a$ для любого $a \in A$. Кольцо $A$ называется локальным, если $A / J(A)$ есть тело. Модуль $M$ называется конечномерным, если $M$ не содержит бесконечных прямых сумм ненулевых модулей.

Мы разобьем доказательство теоремы 1 на ряд утверждений, некоторые из которых представляют самостоятельный интерес. Эти утверждения сгруппированы в два раздела. Окончание доказательства теоремы 1 приведено в замечании 1.

\section{2. Малоинъективные и вполне целозамкнутые модули}

Лемма 1. Пусть $\bar{X}$ есть сущуественное расширение модуля X и $Y$ есть модуль.

(1) Если $f$ есть гомоморфизм из $Y$ в $\bar{X}$ и существует такой гомоморфизм $g: Y \rightarrow X$, что $f$ coвnadaem с g на $f^{-1}(X)$, mо $f(Y) \subseteq X$.

(2) Если X есть $Y$-инъективньй модуль, то $f(Y) \subseteq X$ для любого гомоморфизма $f: Y \rightarrow \bar{X}$.

(3) Если $f$ есть эндоморфизм модуля $\bar{X}$ и существует такой эндоморфизм $g$ модуля $X$, что $f$ совпадает с $g$ на $X \cap f^{-1}(X)$, то $f(X) \subseteq X$.

(4) Если $X \oplus Y$ есть квазинепрерывный модуль, то все его прямые слагаемыле квазинепрерывны, $X$ есть $Y$-инъективный модуль, и $f(Y) \subseteq X$ для любого гомоморфизма $f: Y \rightarrow \bar{X}$.

Доказательство. Докажем п. 1. Допустим, что

$$
x^{*}=(f-g)(x) \in X \cap(f-g)(Y), \quad x \in Y .
$$

Тогда

$$
\begin{gathered}
f(x)=(f-g)(x)+g(x)=x^{*}+g(x) \in X, \quad x \in f^{-1}(X), \\
x^{*} \in(f-g)\left(f^{-1}(X)\right)=0, \quad X \cap(f-g)(Y)=0 .
\end{gathered}
$$

Так как $\bar{X}$ есть существенное расширение модуля $X$, получаем $(f-g)(Y)=0$. Поэтому $f(Y)=g(Y) \subseteq X$.

Докажем п. 2. Так как $f\left(f^{-1}(X)\right) \subseteq X$ и $X$ есть $Y$-инъективный модуль, то существует такой гомоморфизм $g: Y \rightarrow X$, что $g$ совпадает с $f$ на $f^{-1}(X)$. В силу п. $1, f(Y) \subseteq X$.

Утверждение п. 3 следует из п. 1.

Докажем п. 4. Непосредственно проверяется, что все прямые слагаемые квазинепрерывных модулей квазинепрерывны. В силу п. 2, остается доказать, что $X$ есть $Y$-инъективный модуль. Пусть $u: Y \rightarrow X \oplus Y$ есть естественное вложение, $\pi: X \oplus Y \rightarrow X$ есть проекция с ядром $Y, Y_{1}$ есть подмодуль в $Y$, и $f_{1}: Y_{1} \rightarrow X$ есть гомоморфизм. Существует такой эндоморфизм $g_{1}$ модуля $X \oplus Y_{1}$, что $g_{1}\left(x+y_{1}\right)=x+f_{1}\left(y_{1}\right)$. Тогда $g_{1}=g_{1}^{2}$. Так как модуль $X \oplus Y$ квазинепрерывен, то $g_{1}$ продолжается до некоторого эндоморфизма $g$ модуля $X \oplus Y$. Гомоморфизм $\pi g u: Y \rightarrow X$ есть искомое продолжение гомоморфизма $f_{1}$.

Лемма 2. Пусть X есть малоинъективный модуль, $\bar{X}$ есть существенное расширение модуля $X, u f$ есть эндоморфизм модуля $\bar{X}$. Пусть

$$
N=\{x \in X \mid f(x) \in X\} .
$$


(1) Если $f(N) \subseteq N$, то $f(X) \subseteq X$.

(2) Если $f^{2}(m) \in X$ для любого такого элемента $x \in X$, что $f(x) \in X$, то $f(X) \subseteq X$.

(3) Если $\left(f^{2}-f\right)(N) \subseteq X$, то $f(X) \subseteq X$.

(4) Если $\left(f^{2}-f\right)(X) \subseteq X$, то $f(X) \subseteq X$.

Доказательство. Докажем п. 1. Поскольку $f(N) \subseteq N$ и $X$ малоинъективен, то $(f-g)(N)=0$ для некоторого эндоморфизма $g$ модуля $X$. Теперь п. 1 следует из п. 3 леммы 1.

Утверждение п. 2 следует из п. 1.

Докажем п. 3. Пусть $x \in N$. Тогда $f(x) \in X$. По условию, $\left(f^{2}-f\right)(x) \in X$. Поэтому

$$
f^{2}(x)=\left(f^{2}-f\right)(x)+f(x) \in X .
$$

В силу п. 2, $f(X) \subseteq X$.

Утверждение п. 4 следует из п. 3.

Лемма 3. Каждый вполне иุелозамкнутый модуль малоинъективен, каждый малоинъективный модуль квазинепрерывен, причем неразложимые квазинепрерывные модули совпадают с равномерными модулями.

Доказательство. Непосредственно проверяется, что неразложимые квазинепрерывные модули совпадают с равномерными модулями.

Допустим, что $M$ есть вполне целозамкнутый модуль, $X$ есть подмодуль в $M$, и $f$ есть эндоморфизм модуля $X$. Тогда $f$ есть гомоморфизм из $X$ в $M$, переводящий в себя существенный подмодуль $X$ модуля $X$. Так как $M$ есть вполне целозамкнутый модуль, то $f$ продолжается до эндоморфизма $g$ модуля $M$. Поэтому модуль $M$ малоинъективен.

Допустим, что $M$ есть малоинъективный модуль, $E$ есть инъективная оболочка модуля $M, f$ есть идемпотентный эндоморфизм модуля $E$. Так как $\left(f^{2}-f\right)(M)=0 \in M$, то $f(M) \subseteq M$ в силу п. 4 леммы 2.

Лемма доказана.

Лемма 4. Для кольц̧а А равносильны следующие условия:

(1) $x A y=0$ для любых элементов $x, y \in A$ с условием $x y=0$;

(2) в кольце A все правые аннуляторы являются идеалами;

(3) в кольце А все левые аннуляторы являются идеалами.

Утверждения леммы 4 проверяются непосредственно.

Лемма 5. Для кольца А равносильны следующие условия:

(1) А есть инвариантное слева кольщуо;

(2) в кольце A все правые аннуляторы являются идеалами и каждый эндоморфизм любого главного правого идеала хА продолжается до эндоморфизма модуля $A_{A}$.

Следовательно, если А есть малоинъективное справа и слева кольцьо и $x A y=0$ для любых элементов $x, y \in A$ с условием $x y=0$, то А есть инвариантное справа и слева кольичо. 
Доказательство. Докажем, что из условия 1 следует условие 2. По лемме 4, $r(x)$ есть идеал кольца $A$. Пусть $f$ есть эндоморфизм модуля $x A_{A}$. Тогда $f(x)=x b$ для некоторого $b \in A$. Поэтому $f(x c)=x b c$ для любого элемента $c \in A$. Так как кольцо $A$ инвариантно слева, получаем, что $x b=d x$ для некоторого элемента $d \in A$. Определим эндоморфизм $g$ модуля $A_{A}$ правилом $g(a)=d a$. Для любого элемента $c \in A$ справедливы равенства

$$
g(x c)=d x c=x b c=f(x c) .
$$

Поэтому $g$ совпадает с $f$ на $x A$.

Докажем, что из условия 2 следует условие 1 . Пусть $x$ и $a$ есть два элемента кольца $A$. Поскольку $r(x)$ есть идеал кольца $A$, получаем, что $r(x) \subseteq r(x a)$. Поэтому существует такой гомоморфизм $f: x A \rightarrow x a A \subseteq x A$, что $f(x)=x a$. По условию, эндоморфизм $f$ модуля $x A$ продолжается до эндоморфизма $g$ модуля $A_{A}$. Введем обозначение $b=g(1)$. Тогда $x a=g(1 \cdot x)=g(1) x=b x$. Поэтому кольцо $A$ инвариантно слева.

Лемма доказана.

Лемма 6. Пусть М есть модуль и все его фактормодули квазинепрерывньл.

(1) Если все фактормодули модуля $M$ неразложимы, то $M$ есть ичепной модуль.

(2) Если М есть цุиклический модуль над локальным кольцุом, то М есть цุепной модуль.

Доказательство. Докажем утверждение 1. Пусть $X$ и $Y$ есть подмодули в $M, X \nsubseteq Y$, и $h: M \rightarrow M /(X \cap Y)$ есть естественный эпиморфизм. Неразложимый квазинепрерывный модуль $h(M)$ равномерен. Кроме того, $h(X) \cap h(Y)=h(0)$ и $h(X) \neq h(0)$. Тогда $h(Y)=h(0)$ и $Y=X \cap Y \subseteq X$.

Докажем утверждение 2. Так как все фактормодули циклических модулей цикличны, то утверждение 2 вытекает из уже доказанного утверждения 1 и того, что все циклические модули над локальными кольцами неразложимы.

Лемма доказана.

Лемма 7 ([1]). Модуль М является самочнъективным в точности тогда, когда М есть вполне инвариантный подмодуль своей инъективной оболочки.

Лемма 8. Пусть М есть существенное расширение вполне цеелозамкнутого модуля $Х$.

(1) $f(X) \subseteq X$ для любого эндоморфизма $f$ модуля $M$, переводящего в себя некоторый существенный подмодуль модуля $X$.

(2) Если $f$ есть эндоморфизм модуля $M$ и $\operatorname{Ker}(f)$ есть существенньй подмодуль в $M$, mo $f(X) \subseteq X$.

(3) Если $X$ есть существенное расширение некоторого модуля $Y$, который вполне инвариантен в $M$, то $X$ является вполне инвариантным подмодулем в $M$.

(4) Если $X$ есть существенное расширение самоинъективного модуля $Y$, то $X$ является самоинъективным вполне инвариантным подмодулем в $M$.

(5) Если $X$ есть существенное расширение полупростого модуля, то $X$ является самоинъективным вполне инвариантным подмодулем в $М$. 
Доказательство. Докажем п. 1. По условию $f(Y) \subseteq Y$ для некоторого существенного подмодуля $Y$ в $X$. Обозначим через $X_{1}$ модуль $X \cap f^{-1}(X)$ и через $f_{1} \in \operatorname{Hom}\left(X_{1}, X\right)$ ограничение гомоморфизма $f$ на модуль $X_{1}$. Так как $Y \subseteq X_{1}$ и $f_{1}(Y)=f(Y) \subseteq Y$, гомоморфизм $f_{1}: X_{1} \rightarrow X$ переводит в себя существенный подмодуль вполне целозамкнутого модуля $X$. Поэтому $f_{1}$ продолжается до гомоморфизма $g: X \rightarrow X$. Согласно п. 3 леммы 1 , справедливо включение $f(X) \subseteq X$.

Докажем п. 2. Положим $Y=X \cap \operatorname{Ker}(f)$. Тогда $Y$ есть существенный подмодуль в $X$ и $f(Y)=0 \subseteq Y$. Согласно п. $1, f(X) \subseteq X$.

Утверждение п. 3 следует из п. 1.

Докажем п. 4. Пусть $M^{\prime}$ есть инъективная оболочка модуля $M$. Тогда $M^{\prime}$ есть существенное расширение модуля $Y$, поскольку $M^{\prime}$ есть существенное расширение модуля $M, M$ есть существенное расширение модуля $X$, и $X$ есть существенное расширение модуля $Y$. Согласно лемме 7, $Y$ есть вполне инвариантный подмодуль в $M$ и $M^{\prime}$. Согласно п. $3, X$ есть вполне инвариантный подмодуль в $M$ и $M^{\prime}$. Согласно лемме 7 , модуль $X$ самоинъективен.

Все полупростые модули самоинъективны, поэтому п. 5 следует из п. 4.

Лемма 9. Пусть $X$ есть модуль с инъективной оболочкой $\bar{X}, \operatorname{sg}(\bar{X})$ есть идеал кольца $\operatorname{End}(\bar{X})$, образованный всеми эндоморфизмами, ядра которых являются существенными подмодулями в $\bar{X}$. Равносильнь следующче условия:

(1) Х есть вполне ияелозамкнутый модуль;

(2) $f(X) \subseteq X$ для любого эндоморфизма $f$ модуля $\bar{X}$, переводящего в себя некоторый существенный подмодуль модуля $X$;

(3) $X$ есть малоинъективный модуль и $h(X) \subseteq X$ для любого $h \in \operatorname{sg}(\bar{X})$;

(4) X есть малоинъективный модуль, являющийся конечной прямой суммой вполне целозамкнутых модулей.

Доказательство. Импликация (1) $\Rightarrow$ (4) следует из леммы 3.

Докажем, что (4) $\Rightarrow$ (3). По соображениям индукции можно считать, что $X=X_{1} \oplus X_{2}$, где модули $X_{1}$ и $X_{2}$ вполне целозамкнуты. По лемме 3 , модуль $X$ квазинепрерывен. Пусть $\bar{X}$ есть инъективная оболочка модуля $X$. Тогда $\bar{X}=\bar{X}_{1} \oplus \bar{X}_{2}$, где $\bar{X}_{1}$ и $\bar{X}_{2}$ есть инъективные оболочки модулей $X_{1}$ и $X_{2}$ соответственно. Пусть $h \in \operatorname{sg}(\bar{X}), u_{1}: \bar{X}_{1} \rightarrow \bar{X}$ и $u_{2}: \bar{X}_{2} \rightarrow \bar{X}$ есть естественные вложения, $\pi_{1}: \bar{X} \rightarrow \bar{X}_{1}$ и $\pi_{2}: \bar{X} \rightarrow \bar{X}_{2}$ есть естественные проекции. Пусть

$$
\begin{array}{ll}
h_{11}=\pi_{1} h u_{1} \in \operatorname{End}\left(\bar{X}_{1}\right), & h_{22}=\pi_{2} h u_{2} \in \operatorname{End}\left(\bar{X}_{2}\right), \\
h_{12}=\pi_{2} h u_{1} \in \operatorname{Hom}\left(\bar{X}_{1}, \bar{X}_{2}\right), & h_{21}=\pi_{1} h u_{2} \in \operatorname{Hom}\left(\bar{X}_{2}, \bar{X}_{1}\right) .
\end{array}
$$

Так как модули $X_{1}$ и $X_{2}$ вполне целозамкнуты, то по условию (4)

$$
h_{11}\left(X_{1}\right) \subseteq X_{1}, \quad h_{22}\left(X_{2}\right) \subseteq X_{2} .
$$

Кроме того, согласно п. 4 леммы 1 ,

$$
h_{21}\left(X_{2}\right) \subseteq X_{1}, \quad h_{12}\left(X_{1}\right) \subseteq X_{2}
$$

Поэтому

$$
h(X)=h\left(X_{1} \oplus X_{2}\right)=h_{11}\left(X_{1}\right)+h_{12}\left(X_{1}\right)+h_{22}\left(X_{2}\right)+h_{21}\left(X_{2}\right) \subseteq X .
$$


Докажем, что $(3) \Rightarrow(1)$. Пусть $Y_{1}$ есть подмодуль модуля $X$ и $f_{1}$ переводит в себя некоторый существенный подмодуль в $Y_{1}$. Тогда $f_{1}\left(Y_{2}\right) \subseteq Y_{2}$ для некоторого существенного подмодуля $Y_{2}$ модуля $Y_{1}$. Существует такой подмодуль $Z$ в $X$, что $Y_{1} \cap Z=0$ и $X$ есть существенное расширение модуля $Z \oplus Y_{1}$. Непосредственно проверяется, что $X$ есть существенное расширение модуля $Z \oplus Y_{2}$, поскольку $Y_{1}$ есть существенное расширение модуля $Y_{2}$. Поэтому $\bar{X}$ есть существенное расширение модуля $Z \oplus Y_{2}$. Обозначим через $f_{2}$ такой гомоморфизм из $Z \oplus Y_{1}$ в $X$, что $f_{2}\left(z+y_{1}\right)=f_{1}\left(y_{1}\right)$ для любых элементов $z \in Z$ и $y_{1} \in Y_{1}$. Обозначим через $f_{3}$ такой эндоморфизм модуля $Z \oplus Y_{2}$, что $f_{3}\left(z+y_{2}\right)=f_{1}\left(y_{2}\right) \in Y_{2} \subseteq Z \oplus Y_{2}$ для любых элементов $z \in Z$ и $y_{2} \in Y_{2}$. Так как модуль $\bar{X}$ малоинъективен, то эндоморфизм $f_{3}$ продолжается до эндоморфизма $f_{4}$ модуля $X$. Гомоморфизм $f_{2}$ и эндоморфизм $f_{4}$ продолжаются до эндоморфизмов $f$ и $f^{\prime}$ инъективного модуля $\bar{X}$. Обозначим $h=f-f^{\prime} \in \operatorname{End}(\bar{X})$. Так как

$$
h\left(Z \oplus Y_{2}\right)=\left(f-f^{\prime}\right)\left(Z \oplus Y_{2}\right)=0
$$

и $\bar{X}$ есть существенное расширение модуля $Z \oplus Y_{2}$, по условию $h(X) \subseteq X$. Тогда

$$
f(X) \subseteq h(X)+f^{\prime}(X)=h(X)+f_{4}(X) \subseteq X,
$$

причем $f$ совпадает с $f_{1}$ на модуле $Y_{1}$. Поэтому $f_{1}$ продолжается до эндоморфизма модуля $X$ и $X$ есть вполне целозамкнутый модуль.

Утверждение (1) $\Rightarrow$ (2) следует из п. 1 леммы 8.

Докажем, что (2) $\Rightarrow(3)$. Пусть $Y_{1}$ есть подмодуль модуля $X$ и $f_{1} \in \operatorname{End}\left(Y_{1}\right)$. Существует такой подмодуль $Z$ в $X$, что $Y_{1} \cap Z=0$ и $X$ есть существенное расширение модуля $Z \oplus Y_{1}$. Обозначим через $f_{2}$ такой эндоморфизм модуля $Z \oplus Y_{1}$, что $f_{2}\left(z+y_{1}\right)=f_{1}\left(y_{1}\right)$ для любых элементов $z \in Z$ и $y_{1} \in Y_{1}$. Эндоморфизм $f_{2}$ продолжается до эндоморфизма $f$ инъективного модуля $\bar{X}$. Так как $f\left(Z \oplus Y_{1}\right) \subseteq Z \oplus Y_{1}$, получаем, что $f$ переводит в себя некоторый существенный подмодуль в $X$. По условию, $f(X) \subseteq X$. Тогда модуль $X$ малоинъективен, поскольку $f_{1}$ продолжается до эндоморфизма модуля $X$. Пусть $h$ есть эндоморфизм модуля $\bar{X}$ и $\operatorname{Ker}(h)$ является существенным подмодулем в $\bar{X}$. Пусть $Y=X \cap \operatorname{Ker}(h)$. Так как $Y$ есть существенный подмодуль в $X$ и $h(Y)=0 \subseteq Y$, мы видим, что $h$ переводит в себя некоторый существенный подмодуль в $X$. По условию, $h(X) \subseteq X$.

Лемма доказана.

Лемма 10. Пусть $X$ есть самоинъективный модуль и $R=\operatorname{End}(X)$.

(1) $\operatorname{sg}(X)=J(R), R / J(R)$ есть регулярное кольцо, и все его идемпотенты поднимаются до идемпотентов кольиа $R$.

(2) Если X есть неразложимый модуль, то X есть равномерный модуль с локальным кольиом эндоморфизмов.

(3) Если А есть самоинъективное справа кольцо, то при естественном изоморфизме $A \rightarrow \operatorname{End}\left(A_{A}\right)$ идеал $J(A)$ переходит на идеал $\operatorname{sg}\left(A_{A}\right)$.

Доказательство. Утверждение 1 доказано в [1]. Утверждение 2 вытекает из утверждения 1 и леммы 3. Утверждение 3 вытекает из утверждения 1.

Лемма 11. Пусть $X$ есть малоинъективный модуль, $\bar{X}$ есть инъективная оболочка модуля $X, u \operatorname{sg}(X)$ есть идеал кольиа $\operatorname{End}(X)$, образованный всеми эндоморфизмами, ядра которых являются существенными подмодулями в $X$. 
(1) Если $\alpha(Z) \subseteq Z$ для любого $\alpha \in \operatorname{sg}(X)$ и каждого сущчественного подмодуля $Z$ в $X$, то $X$ есть вполне целозамкнутый модуль.

(2) Если каждый существенньй подмодуль $Y$ в $X$ вполне инвариантен в $X$, то $X$ является вполне иелозамкнутым модулем. В частности, если X есть инвариантный модуль, то Х есть вполне целозамкнутый модуль.

(3) Если $\operatorname{sg}(X)=0$, то X есть вполне изелозамкнутый модуль.

(4) Если модуль X несингулярен, то X есть вполне цุелозамкнутый модуль.

(5) Если X есть конечная прямая сумма инвариантных модулей, то X есть вполне целозамкнутый модуль.

(6) Если $X$ есть ичепной модуль и $\alpha$ есть автоморфизм модуля $\bar{X}$ с условием $\alpha(X) \nsubseteq X$, mo $\alpha^{-1}(X) \subseteq X$.

(7) Если $X$ есть ичепной модуль $и \operatorname{sg}(X) \subseteq J(\operatorname{End}(X))$, то X есть вполне целозамкнутый модуль.

Доказательство. Докажем утверждение 1. Пусть $h \in \operatorname{sg}(\bar{X})$. Согласно лемме 9, достаточно доказать, что $h(X) \subseteq X$. Обозначим через $Y$ сумму всех подмодулей в $X$, переходящих в себя под действием $h$. Так как $X \cap \operatorname{Ker}(h) \subseteq Y$, мы видим, что $Y$ есть существенный подмодуль в $X$. Поскольку $h(Y) \subseteq Y$ и модуль $X$ малоинъективен, получаем, что $(h-g)(Y)=0$ для некоторого эндоморфизма $g$ модуля $X$. Так как $g(X \cap \operatorname{Ker}(h))=0$, находим, что $g \in \operatorname{sg}(X)$. Пусть

$$
\begin{aligned}
Z & =\{x \in X \mid(h-g)(x) \in Y\}, \\
Z^{*} & =\{x \in X \mid h(x) \in X\} .
\end{aligned}
$$

Так как $h(Z) \subseteq(h-g)(Z)+g(Z) \subseteq X$, получаем, что $Z \subseteq Z^{*} \subseteq X$. Кроме того, $Y \subseteq Z, Z$ есть существенный подмодуль в $X$, и $(h-g)(Z) \subseteq Y \subseteq Z$. Существует такой гомоморфизм $\beta^{*}: Z^{*} / Y \rightarrow X$, что $\beta^{*}\left(z^{*}+Y\right)=(h-g)\left(z^{*}\right) \in X$. Тогда

$$
Z / Y=\left\{z^{*}+Y \in Z^{*} / Y \mid \beta^{*}\left(z^{*}+Y\right) \in Y\right\} .
$$

Так как $Y$ есть существенный подмодуль в $X$, получаем, что $Z / Y$ есть существенный подмодуль в $Z^{*} / Y$. Так как модуль $X$ малоинъективен и $(h-g)(Z) \subseteq Z$, получаем, что $(h-g-f)(Z)=0$ для некоторого эндоморфизма $f$ модуля $X$. Так как $f(Y)=(h-g)(Y)=0$, мы видим, что $f \in \operatorname{sg}(X)$. Следовательно, $f+g \in \operatorname{sg}(X)$. По условию, $(g+f)(Z) \subseteq Z$. Кроме того, $(h-g-f)(Z)=0$. Поэтому $h(Z)=(g+f)(Z) \subseteq Z$. Так как $Y$ есть сумма всех подмодулей в $X$, переходящих в себя под действием $h$, заключаем, что $Z \subseteq Y \subseteq Z$ и $Z=Y$. Кроме того, $Z / Y$ есть существенный подмодуль в $Z^{*} / Y$. Поэтому $Z^{*} / Y=0$ и $Z^{*}=Y$. Согласно п. 3 леммы $1, h(X) \subseteq X$.

Утверждения 2 и 3 вытекают из утверждения 1.

Утверждение 4 вытекает из утверждения 3 и того, что $\operatorname{sg}(X)=0$ для несингулярного модуля $X$.

Утверждение 5 вытекает из утверждения 2 и леммы 9.

Докажем утверждение 6. Введем обозначение

$$
N=\left\{x \in X \mid \alpha^{-1}(x) \in X\right\} .
$$


Тогда

$$
\alpha^{-1}(N)=\{x \in X \mid \alpha(x) \in X\} .
$$

По п. 1 леммы 2, примененной к $\alpha^{-1}$, достаточно доказать, что $\alpha^{-1}(N) \subseteq N$. Допустим, что $\alpha^{-1}(N) \nsubseteq N$. Так как $X$ есть цепной модуль, то $N \subseteq \alpha^{-1}(N)$. Тогда $\alpha\left(\alpha^{-1}(N)\right) \subseteq \alpha^{-1}(N)$. Из п. 1 леммы 2, примененного к $\alpha$, вытекает, что $\alpha(X) \subseteq X$. Получено противоречие.

Докажем утверждение 7. Пусть $h \in \operatorname{sg}(\bar{X})$ и $K=X \cap \operatorname{Ker}(h)$. По лемме 9, достаточно доказать, что $h(X) \subseteq X$. Согласно п. 1 леммы 10,

$$
\operatorname{sg}(\bar{X})=J(\operatorname{End}(\bar{X})) .
$$

Поэтому $1-h$ есть автоморфизм модуля $\bar{X}$ и действует тождественно на существенном подмодуле $K$ модуля $X$. Тогда автоморфизм $(1-h)^{-1}$ модуля $\bar{X}$ действует тождественно на существенном подмодуле $K$ модуля $X$. Если $(1-h)(X) \subseteq X$, то $h(X) \subseteq X+(1-h)(X) \subseteq X$, и все доказано.

Допустим, что $(1-h)(X) \nsubseteq \subseteq X$. Согласно утверждению $6,(1-h)^{-1}(X) \subseteq X$. Поэтому существует такой эндоморфизм $f$ модуля $X$, что $f(x)=(1-h)^{-1}(x)$ для всех $x \in X$. Кроме того, $(1-f)(K)=0$, поскольку $(1-h)^{-1}$ действует тождественно на $K$. Поэтому $1-f \in \operatorname{sg}(X)$. Кроме того, $\operatorname{sg}(X) \subseteq J(\operatorname{End}(X))$ по условию. Поэтому $f=1-(1-f)$ есть автоморфизм модуля $X$. Поскольку $f(x)=(1-h)^{-1}(x)$ для всех $x \in X$, заключаем, что $(1-h)^{-1}(X)=X$. Поэтому $X=(1-h)(X)$. Получено противоречие.

Лемма доказана.

Лемма 12 ([1]). Пусть $A$ есть кольцуо и $Q$ есть его максимальное правое кольцо частных. Если модуль $Q_{A}$ является инъективной оболочкой модуля $A_{A}$, то отображение $\varphi: \operatorname{End}\left(Q_{A}\right) \rightarrow Q$, при котором $\varphi(f)=f(1)$, является изоморфизмом колеи.

Лемма 13. Если А есть несингулярное справа кольцьо и $Q$ есть его максимальное правое кольичо частных, то равносильны следующчие условия:

(1) $A_{A}$ есть вполне ичелозамкнутый модуль;

(2) А есть вполне ичелозамкнутое справа подкольцьо в $Q$;

(3) $A_{A}$ есть малоинъективньй модуль.

Доказательство. Так как кольцо $A$ несингулярно справа, мы видим, что $Q_{A}$ есть инъективная оболочка модуля $A_{A}[1]$. Согласно лемме 12 , кольцо $Q$ можно естественным образом отождествить с кольцом $\operatorname{End}\left(Q_{A}\right)$. Поэтому эквивалентность $(1) \Longleftrightarrow(2)$ вытекает из леммы 9.

Эквивалентность (1) $\Longleftrightarrow$ (3) вытекает из леммы 3 и п. 4 леммы 11.

Лемма доказана.

Лемма 14. Для кольц̧а А равносильны следующче условия:

(1) А есть несингулярное справа кольио без нетривиальных идемпотентов, и $A_{A}$ есть вполне иелозамкнутый модуль;

(2) А есть несингулярное справа кольијо без нетривиальных идемпотентов, и $A_{A}$ есть малоинъективный модуль;

(3) А есть равномерная справа область, и $A_{A}$ есть вполне целозамкнутый модуль; 
(4) А есть инвариантная слева область, А имеет классическое двустороннее тело частных $Q$, причем А есть вполне иелозамкнутое справа подкольцо в $Q$ и классически вполне цчелозамкнутое справа подкольцьо в $Q$.

Доказательство. Эквивалентность (1) леммы 13.

Импликация (4) $\Rightarrow$ (3) вытекает из леммы 3.

Импликация (3) $\Rightarrow$ (1) очевидна.

Докажем, что $(1) \Rightarrow(3)$. Так как кольцо $A$ не имеет нетривиальных идемпотентов, то $A_{A}$ есть вполне целозамкнутый неразложимый модуль. По лемме 3 , кольцо $A$ равномерно справа. Непосредственно проверяется, что несингулярное справа, равномерное справа кольцо $A$ является областью.

Докажем, что (3) $\Rightarrow(4)$. Так как $A$ есть равномерная справа область, мы заключаем, что $A$ имеет классическое правое тело частных $Q$. По лемме 5 , область $A$ инвариантна слева. Тогда область $A$ равномерна слева и имеет классическое левое тело частных. Поэтому классическое правое тело частных $Q$ является двусторонним телом частных области $A$. По лемме $13, A$ является вполне целозамкнутым справа подкольцом в $Q$.

Пусть $0 \neq q \in Q, 0 \neq a \in A$, и $q^{i} a \in A$ для всех натуральных чисел $i$. Обозначим через $B$ существенный правый идеал $\sum_{i=1}^{\infty} q^{i} a A$ равномерной области $A$. Так как $q B \subseteq B$ и $A$ есть вполне целозамкнутое справа подкольцо в $Q$, получаем, что $q A \subseteq A$ и $q \in A$. Поэтому $A$ является классически вполне целозамкнутым справа подкольцом в $Q$.

Лемма доказана.

Лемма 15. Для модуля X равносильнь следующче условия:

(1) X есть вполне цеелозамкнутый неразложимьй модуль;

(2) Х есть равномерный модуль, и либо Х есть самоинъективный модуль с локальным кольияом $\operatorname{End}(X)$ и $\operatorname{sg}(X)=J(\operatorname{End}(X))$, либо $X$ есть малоинъективныий модуль $u$ каждый его ненулевой эндоморфизм является мономорфизмом.

Доказательство. Пусть $M$ есть инъективная оболочка модуля $X$.

Докажем, что (1) $\Rightarrow(2)$. По лемме $3, X$ есть малоинъективный равномерный модуль. Если каждый ненулевой эндоморфизм модуля $X$ является мономорфизмом, то все доказано.

Допустим, что существует ненулевой эндоморфизм $g \in \operatorname{End}(X)$ с ненулевым ядром. Эндоморфизм $g$ продолжается до ненулевого эндоморфизма $h \in \operatorname{sg}(M)$. Обозначим через $Y$ ненулевой вполне инвариантный подмодуль $\sum_{f \in \operatorname{sg}(M)} f(M)$ инъективного равномерного модуля $M$. По лемме $9, Y \subseteq X$; согласно п. 3 леммы $8, X$ есть вполне инвариантный подмодуль в $M$. По лемме 7 , модуль $X$ самоинъективен. Так как $X$ есть неразложимый самоинъективный модуль, согласно п. 2 леммы $10 \operatorname{End}(X)$ есть локальное кольцо и $\operatorname{sg}(X)=J(\operatorname{End}(X))$.

Докажем, что (2) $\Rightarrow(1)$. Без ограничения общности можно считать, что $X$ есть малоинъективный модуль и каждый его ненулевой эндоморфизм является мономорфизмом. Согласно п. 3 леммы 11, $X$ есть вполне целозамкнутый модуль.

Лемма доказана.

Лемма 16. Пусть А есть кольц̧о и $N_{l}(A)$ есть множество всех его левых делителей нуля. Равносильны следуюшие условия:

5 Дискретная математика, т.23 №3 
(1) кольцуо А не имеет нетривиальных идемпотентов, и $A_{A}$ есть вполне цеелозамкнутый модуль;

(2) А есть равномерное справа кольцо, и либо А есть самоинъективное справа локальное кольияо и

$$
J(A)=\operatorname{sg}\left(A_{A}\right)=\operatorname{Sing}\left(A_{A}\right)=N_{l}(A)
$$

либо А есть инвариантная слева область и $A_{A}$ есть малоинъективньй модуль.

Доказательство. Импликация (2) $\Rightarrow(1)$ очевидна.

Докажем, что (1) $\Rightarrow(2)$. По лемме 3 , кольцо $A$ равномерно справа. Поэтому

$$
\operatorname{Sing}\left(A_{A}\right)=N_{l}(A)=\operatorname{sg}\left(A_{A}\right) \text {. }
$$

Остальные утверждения вытекают из лемм 14 и 15.

\section{3. Вполне целозамкнутые циклические модули}

Лемма 17. Пусть А есть область, а есть ее необратимый элемент, причем модуль $A_{A}$ малоинъективен.

(1) $\bigcap_{i=1}^{\infty} a^{i} A=0$.

(2) Если главный правый идеал аА строго содержит некоторый первичный идеал $P$ области $A$, то $P=0$.

Доказательство. Докажем утверждение 1 . Можно считать, что $a \neq 0$. По лемме 14 , область $A$ имеет классическое тело частных $Q$, которое содержит элемент $a^{-1}$. Обозначим через $X$ правый идеал $\bigcap_{i=1}^{\infty} a^{i} A$ области $A$. Существует такой эндоморфизм $f$ модуля $X_{A}$, что $f(x)=a^{-1} x$ для всех $x \in X$. По условию, эндоморфизм $f$ продолжается до некоторого эндоморфизма $g$ модуля $A_{A}$. Введем обозначение $b=g(1) \in A$. Допустим, что $X \neq 0$. Возьмем ненулевой элемент $x \in X$. Тогда $a^{-1} x=f(x)=g(1 \cdot x)=b x$, откуда следует, что $a^{-1}=b \in A$ и элемент $a$ обратим в $A$. Получено противоречие. Поэтому $X=0$.

Докажем утверждение 2. Пусть $h: A \rightarrow A / P$ есть естественный кольцевой эпиморфизм. Так как $P \subsetneq a A$, заключаем, что $h(a) \neq h(0), P=a Y \subseteq Y$ для некоторого идеала $Y$ области $A$ и $h(a) h(Y)=h(0)$. По лемме 5, область $A$ инвариантна слева. Тогда $h(A)$ есть инвариантное слева первичное кольцо. Поэтому $h(A)$ есть область, причем $h(a) h(Y)=h(0)$. Тогда $h(Y)=h(0), Y \subseteq P \subseteq Y$, и $P=Y$. Поэтому $P=a P$ и $P \subseteq \bigcap_{i=1}^{\infty} a^{i} A$. Согласно утверждению $1, P=0$.

Лемма 18. Пусть А есть цеенная справа, малоинъективная справа область. Тогда

(1) А имеет не более двух первичных идеалов;

(2) А есть инвариантная иепная область. 
Доказательство. Согласно лемме 5, область $A$ инвариантна слева.

Докажем утверждение 1. Допустим противное. Тогда инвариантная слева, цепная справа область $A$ имеет ненулевой первичный идеал $P$, строго лежащий в $J(A)$. Пусть $a \in J(A) \backslash P$. Это противоречит п. 2 леммы 17.

Докажем утверждение 2. Так как $A$ есть инвариантная слева, цепная справа область, она является цепной слева областью. Остается доказать, что область $A$ инвариантна справа. Достаточно доказать, что $b a \in a A$ для любых ненулевых элементов $a \in J(A), b \in A$. Допустим, что $b a \notin a A$. Так как $A$ есть цепная справа область, получаем, что $a A \subseteq b a A$. Тогда $a=b a c$ для некоторого ненулевого элемента $c \in A$, причем элемент $c$ необратим в $A$, поскольку $b a \notin a A$. Поэтому $c \in J(A)$ и $a=b^{i} a c^{i}$ для всех натуральных чисел $i$. Тогда $0 \neq a \in \bigcap_{i=1}^{\infty} A c^{i}$.

Так как $A$ есть область и $0 \neq a \in J(A)$, заключаем, что $a \notin A a^{2}$. Поэтому правый идеал $a A$ не лежит в $A a^{2}=A a^{2} A$. Пусть $h: A \rightarrow A a^{2}$ есть естественный кольцевой эпиморфизм. Так как $h(J(A))$ есть единственный первичный идеал кольца $h(A)$, заключаем, что $h(J(A))$ является первичным радикалом кольца $h(A)$. Поэтому $h(c)$ есть нильпотентный элемент кольца $h(A)$. Тогда $c^{n}=x a^{2}$ для некоторых $n \in \mathbf{N}$ и $x \in A$. Так как $0 \neq a \in \bigcap_{i=1}^{\infty} A c^{i}$, получаем, что $0 \neq a \in A x a^{2}$. Поэтому $1 \in A a$, получаем противоречие. Лемма доказана.

Лемма 19. Пусть А есть инвариантная цеепная область и $Q$ есть ее классическое тело частных. Если $q \in Q \backslash A$, то $q^{-1} \in$ A. Кроме того, равносильны следующие условия:

(1) А есть классически вполне цуелозамкнутое справа подкольцо в $Q$;

(2) модуль $A_{A}$ малоинъективен;

(3) $\bigcap_{i=1}^{\infty} a^{i} A=0$ для любого необратимого элемента а области $A$;

(4) А имеет не более двух первичных идеалов.

Доказательство. Пусть $q=a b^{-1} \in Q \backslash A$, где $a$ и $b$ есть ненулевые элементы цепной области $A$. Так как $a b^{-1} \notin A$, то $a \notin A b$. Поэтому $b \in A a$ и $q^{-1}=b a^{-1} \in A$.

Импликация (1) $\Rightarrow$ (2) следует из леммы 14.

Импликация (2) $\Rightarrow$ (3) следует из п. 1 леммы 17.

Докажем, что (1) $\Rightarrow$ (4). Допустим противное. Тогда инвариантная цепная область $A$ имеет ненулевой первичный идеал $X$, строго лежащий в $J(A)$. Первичный идеал $X$ инвариантного кольца $A$ вполне первичен. Пусть $a \in J(A) \backslash X$. Так как идеал $X$ инвариантного цепного кольца $A$ не содержит идеал $a A$, получаем, что главный идеал $a A$ строго содержит вполне первичный идеал $X$. Поэтому $X=a X$. Тогда $X=a^{i} X$ для всех $i$. Поэтому $X \subseteq \bigcap_{i=1}^{\infty} a^{i} A=0$ и $X=0$. Получено противоречие.

Докажем, что (4) $\Rightarrow(3)$. Пусть $а$ есть необратимый элемент инвариантной цепной области $A, X=\bigcap_{i=1}^{\infty} a^{i} A$, и $h: A \rightarrow A / X$ есть естественный эпиморфизм. Тогда $a$ есть элемент первичного идеала $J(A)$ области $A$. Допустим, что $X \neq 0$. Так как 0 есть первичный идеал и $A$ имеет не более двух первичных идеалов, то кольцо $h(A)$ имеет ровно один первичный идеал $h(J(A))$, который является первичным радикалом кольца $h(A)$. Так как первичный радикал является нильидеалом, элемент $h(a)$ нильпотентен. Поэтому $a^{n} \in X$ для некоторого натурального числа $n$. Тогда $a^{n}=a^{n+1} b$ для некоторого элемента $b$ области $A$. Поэтому $1=a b$ и элемент $a$ обратим. Получено противоречие. 
Докажем, что (3) $\Rightarrow(1)$. Пусть $0 \neq q \in Q, 0 \neq a \in A$, и $q^{i} a \in A$ для всех натуральных чисел $i$. Допустим, что $q \notin A$. Тогда $q^{-1}=b$ есть необратимый элемент области $A$. Пусть $Y$ есть ненулевой идеал $\sum_{i=1}^{\infty} q^{i} a$ области $A$. Так как $q Y \subseteq Y$ и $q^{-1}=b \in A$, получаем, что $Y \subseteq b Y \subseteq Y, Y=b Y$, и $Y=b^{i} Y$ для всех $i$. Поэтому $Y \subseteq \bigcap_{i=1}^{\infty} b^{i} A=0$. Получено противоречие.

Лемма доказана.

Лемма 20. Для кольиа А равносильны следующие условия:

(1) А есть цепная справа область, причем $A_{A}$ есть вполне целозамкнутый модуль;

(2) А есть цепная слева область, причем $A_{A}$ есть вполне целозамкнутый модуль;

(3) А есть цепная справа область, причем модуль $A_{A}$ малоинъективен;

(4) А есть иепная слева область, причем модуль $A_{A}$ малоинъективен;

(5) А есть инвариантная цепная область, имеющая не более двух первичных идеалов.

Доказательство. Эквивалентности (1)

Импликация (3) $\Rightarrow$ (5) следует из леммы 18.

Импликация (5) $\Rightarrow$ (4) следует из леммы 19.

Докажем, что (4) $\Rightarrow(3)$. Пусть $a$ и $b$ есть два ненулевых элемента области $A$. Так как по лемме 3 область $A$ равномерна справа, заключаем, что $a c=b d \neq 0$ для некоторых ненулевых элементов $c, d \in A$. Так как $A$ есть цепная слева область, видим, что либо $c=x d$, либо $d=y c$ для некоторых $x, y \in A$. Поэтому либо $a x d=b d$, либо $a c=b y c$. Так как $A$ есть область, получаем, что либо $b=a x$, либо $a=b y$. Поэтому $A$ является цепной справа областью.

Лемма доказана.

Лемма 21. Для кольиа А равносильны следующие условия:

(1) А есть цеепное справа, малоинъективное справа кольцо;

(2) либо А есть инвариантная ичепная область, имеющая не более двух первичных идеалов, либо А есть самоинъективное справа, иепное справа и слева кольио.

Доказательство. Импликация (2) $\Rightarrow$ (1) следует из леммы 20.

Докажем, что $(1) \Rightarrow(2)$. Если $A$ есть область, то по лемме $20 A$ есть инвариантная цепная область, имеющая не более двух первичных идеалов. Допустим, что $A$ не область. Так как $A$ есть цепное справа кольцо и $A=\operatorname{End}\left(A_{A}\right)$, получаем, что $\operatorname{sg}\left(A_{A}\right)=J(A)$. Согласно п. 7 леммы $11, A_{A}$ есть вполне целозамкнутый модуль. По лемме $16, A$ есть самоинъективное справа кольцо.

Пусть $x, y \in A$. Так как $A$ есть цепное справа кольцо, мы видим, что либо $r(y) \subseteq r(x)$, либо $r(x) \subseteq r(y)$. Поэтому либо существует гомоморфизм $f: y A \rightarrow x A$ с условием $f(y)=x$, либо существует гомоморфизм $g: x A \rightarrow y A$ с условием $g(x)=y$. Кроме того, кольцо $A$ самоинъективно справа. Поэтому либо существует элемент $a \in A$ с условием $a y=x$, либо существует элемент $b \in A$ с условием $b x=y$. Поэтому $A$ есть цепное слева кольцо.

Лемма доказана.

Лемма 22 ([7]). Для кольйа А равносильны следующие условия: 
(1) все ичиклические правые А-модули квазинепрерывны;

(2) $A=\prod_{i=1}^{n} A_{i}$, где $A_{i}$ есть либо простое артиново кольцо, либо равномерное справа кольцуо, над которым все циклические правые модули квазинепрерывны, $i=1, \ldots, n$.

При выполнении этих условий каждый цฺиклический правый А-модуль является конечной прямой суммой ичиклических равномерных модулей.

Лемма 23. Для модуля М равносильны следующие условия:

(1) модуль М малопроективен и все его фактормодули малоинъективны;

(2) модуль М малоинъективен и все его подмодули малопроективныл.

Доказательство. Докажем, что $(1) \Rightarrow(2)$. Пусть $N$ есть подмодуль модуля $M, \bar{f}$ есть эндоморфизм фактормодуля $\bar{N}=N / P$, и $h: M \rightarrow M / P$ есть естественный эпиморфизм. Так как модуль $M / P$ малоинъективен, $\bar{f}$ продолжается до эндоморфизма $\bar{g}$ модуля $M / P$. Поскольку модуль $M$ малопроективен, справедливо равенство $\bar{g} h=h g$ для некоторого эндоморфизма $g$ модуля $M$. Поэтому $g(N) \subseteq N$. Тогда $g$ индуцирует эндоморфизм $f$ модуля $N$ и $\bar{f} h_{N}=h_{N} f$, где $h_{N}: N \rightarrow N / P$ есть естественный эпиморфизм. Поэтому модуль $N$ малопроективен.

Докажем, что $(2) \Rightarrow(1)$. Пусть $M / N$ есть фактормодуль модуля $M, \bar{X}$ есть подмодуль модуля $M / N$, и $\bar{f}$ есть эндоморфизм модуля $\bar{X}$. Существует такой подмодуль $X$ модуля $M$, что $N \subseteq X$ и $\bar{X}=X / N$. Пусть $h: X \rightarrow X / N$ есть естественный эпиморфизм. По условию, модуль $X$ малопроективен. Поэтому $\bar{f} h=h f$ для некоторого эндоморфизма $f$ модуля $X$ с условием $f(N) \subseteq N$. Так как модуль $M$ малоинъективен, то $f$ продолжается до некоторого эндоморфизма $g$ модуля $M$. Поскольку $g(N)=f(N) \subseteq N$, мы видим, что $g$ индуцирует эндоморфизм $\bar{g}$ модуля $M / N$. Так как $\bar{g}$ совпадает с $\bar{f}$ на модуле $\bar{X}$, заключаем, что модуль $M / N$ малоинъективен.

Лемма 24. Для кольц̧а А равносильны следующче условия:

(1) все циклические правые А-модули малоинъективны;

(2) А малоинъективно справа и все его правые идеаль являются малопроективными правыми А-модулями;

(3) $A=\prod_{i=1}^{n} A_{i}$, где $A_{i}$ есть либо простое артиново кольцьо, либо равномерное справа кольцо, над которым все цүиклиеские правые модули малоинъективны и конечномерны, $i=1, \ldots, n$;

(4) $A=\prod_{i=1}^{n} A_{i}$, где $A_{i}$ есть либо простое артиново кольцо, либо малоинъективное справа равномерное справа кольцьо, в котором все правые идеалы малопроективны, $i=1, \ldots, n$.

Доказательство. Эквивалентность (1) $A_{A}$ проективен.

Импликация (1) $\Rightarrow$ (3) следует из леммы 22 и того, что по лемме 3 каждый малоинъективный модуль квазинепрерывен. 
Эквивалентность (3)

Докажем, что (3) $\Rightarrow(1)$. Так как над простым артиновым кольцом все модули инъективны, заключаем, что все циклические правые $A_{i}$-модули малоинъективны, $i=1, \ldots, n$. Непосредственно проверяется, что все циклические правые $A$-модули малоинъективны.

Лемма 25 ([5]). Пусть А есть инвариантное ичепное кольцьо, и пусть А имеет ровно один первичный идеал. Тогда все цииклические правые А-модули и все цчиклические левые А-модули квазиинъективны.

Лемма 26. Для кольцุа А равносильны следующие условия:

(1) А есть локальная область, и все ичклические правые А-модули вполне целозамкнуmbl;

(2) А есть локальная область, и все ичиклические левые А-модули вполне целозамкнуты;

(3) А есть локальная область, и все ичиклические правые А-модули малоинъективны;

(4) А есть локальная область, и все циклические левьле A-модули малоинъективнь;;

(5) А есть инвариантная ичепная область, и А имеет не более двух первичных идеалов.

Доказательство. Достаточно доказать эквивалентность условий 1, 3 и 5.

Импликация (1) $\Rightarrow$ (3) следует из леммы 3 .

Докажем, что $(3) \Rightarrow(5)$. Согласно п. 2 леммы $6, A$ есть цепное справа кольцо. Поэтому условие 5 следует из леммы 4.

Докажем, что $(5) \Rightarrow(1)$. Пусть $B$ есть идеал инвариантного кольца $A$. Если $B=0$, то $A / B$ по лемме 4 есть вполне целозамкнутый правый модуль над кольцом $A / B$. Если $B \neq 0$, то кольцо $A / B$ имеет ровно один первичный идеал, и $A / B$ по лемме 9 есть вполне целозамкнутый правый модуль над кольцом $A / B$.

Лемма доказана.

Лемма 27 ([2]). Пусть А есть ичепное кольц̧о, и пусть $x A \subseteq A x$ или $A x \subseteq x A$ для любого элемента $x \in A$.

(1) В А каждый первичный идеал вполне первичен.

(2) Если А есть кольияо с условием минимальности для первичных идеалов, то $A$ есть инвариантное кольцьо.

Лемма 28. Пусть А есть локальное кольцо, все ииклические правые А-модули вполне целозамкнуты, и А не область.

(1) А есть самоинъективное справа, иуепное (справа и слева) кольио.

(2) $J x \subseteq x A$ для любого $x \in A$.

(3) $x A \subseteq A x$ или $A x \subseteq x A$ для любого элемента $x \in A$.

(4) А есть инвариантное иепное кольио с ненулевым первичным радикалом $P, A / P$ есть область, и А имеет не более двух первичных идеалов. 
Доказательство. Докажем утверждение 1. Согласно п. 2 леммы 6, $A$ есть цепное справа кольцо. По лемме 21, $A$ есть самоинъективное справа, цепное слева кольцо.

Докажем утверждение 2. Введем обозначение $J=J(A)$. Согласно п. 3 леммы 10 , при естественном изоморфизме $A \rightarrow \operatorname{End}\left(A_{A}\right)$ идеал $J$ переходит на идеал $\operatorname{sg}\left(A_{A}\right)$. Если $0 \neq x \in A$, то инъективный цепной модуль $A_{A}$ есть инъективная оболочка циклического вполне целозамкнутого модуля $x A$, откуда $J x A \subseteq x A$ по лемме 9.

Докажем утверждение 3. Допустим, что $x \in A, x a \notin A x$, и $b x \notin x A$ для некоторых $a, b \in A$. Так как $A$ есть цепное кольцо согласно утверждению 1 , получаем, что

$$
x=c x a=b x d=c b x d a
$$

для некоторых $c, d \in A$. Если элемент $c$ обратим, то $x a=c^{-1} c x a=c^{-1} x \in A x$, и получаем противоречие. Если элемент $d$ обратим, то $b x=b x d d^{-1}=x d^{-1} \in x A$, и получаем противоречие. Поэтому $c, d \in J$ и $x=c b x d a \in J x J$, причем $J x \subseteq x A$. Поэтому $x \in J x J \subseteq x J$ и $x=x y$ для некоторого $y \in J$. Тогда элемент $1-y$ обратим и $x(1-y)=0$, откуда $x=0, x a \in A x$, и получаем противоречие.

Докажем утверждение 4 . Первичный радикал $P$ цепного кольца $A$ является первичным идеалом. Согласно п. 3 и п. 1 леммы 11, первичный идеал $P$ вполне первичен. Тогда $A / P$ есть цепная область, над которой все циклические правые модули вполне целозамкнуты. По лемме $10, A / P$ есть инвариантная цепная область, и $A / P$ имеет не более двух первичных идеалов. Так как каждый первичный идеал кольца $A$ содержит первичный радикал $P$, кольцо $A$ имеет не более двух первичных идеалов. Согласно п. 2 леммы 27, $A$ есть инвариантное кольцо.

Лемма доказана.

Лемма 29. Для кольцฺа А равносильны следующче условия:

(1) А есть локальное кольцьо, и все ициклческие правые А-модули вполне целозамкнуты;

(2) А есть инвариантное ичепное кольио, А имеет не более двух первичных идеалов, и либо А есть область, либо для любого нильпотентного непервичного идеала $B$ кольц̧а А факторкольио А/B самоинъективно справа.

Доказательство. Докажем что (1) $\Rightarrow(2)$. По лемме 26 можно считать, что $A$ не область. Согласно п. 4 леммы 28, $A$ есть инвариантное цепное кольцо с ненулевым первичным радикалом $P, A / P$ есть область, и $A$ имеет не более двух первичных идеалов. Пусть $B$ есть нильпотентный непервичный идеал кольца $A$. Тогда $B$ строго содержится в $P$. Поэтому $A / B$ не область. Так как $A / B$ есть локальное кольцо и все циклические правые модули над $A / B$ вполне целозамкнуты, согласно п. 4 леммы 28 кольцо $A / B$ самоинъективно справа.

Докажем, что (2) $\Rightarrow(1)$. Пусть $B$ есть идеал инвариантного кольца $A$. Достаточно доказать, что факторкольцо $A / B$ является вполне целозамкнутым правым модулем над кольцом $A / B$. Первичный радикал $P$ инвариантного цепного кольца $A$ является вполне первичным идеалом. Тогда $A / P$ есть инвариантная цепная область, и $A / P$ имеет не более двух первичных идеалов. Если $P \subseteq B$, то $A / B$ есть, по лемме 26 , вполне целозамкнутый правый модуль над $A / B$. Допустим, что $B \subsetneq P$ и $p \in P \backslash B$. Так как $A$ есть инвариантное цепное кольцо, очевидно, что $p A$ есть нильпотентный идеал и $B \subset p A$. Тогда $B$ есть нильпотентный непервичный идеал. По условию, кольцо $A / B$ самоинъективно справа. Поэтому $A / B$ является вполне целозамкнутым правым модулем над $A / B$.

Лемма доказана. 
Лемма 30. Для кольцุа А равносильны следующчие условия:

(1) все циклические правые А-модули вполне цчелозамкнуты;

(2) $A=A_{1} \times \ldots \times A_{n}$, и $A_{i}$ есть либо простое артиново кольцо, либо равномерное справа кольцчо, над которым все ичиклические правые модули вполне цчелозамкнуть и конечномерны, $i=1, \ldots, n$;

(3) $A=A_{1} \times \ldots \times A_{n}$, и для любого і верно, что либо $A_{i}$ есть простое артиново кольцุо, либо $A_{i}$ есть инвариантная слева, равномерная справа область, над которой все ииклические правые модули вполне целозамкнуты, либо $A_{i}$ есть инвариантное ценпное кольцо, $A_{i}$ имеет не более двух первичных идеалов, и для любого нильпотентного непервичного идеала В кольца $A_{i}$ факторкольио $A_{i} / B$ самоинъективно справа.

Доказательство. Импликация (3) $\Rightarrow$ (2) вытекает из леммы 29.

Импликация (2) $\Rightarrow$ (1) проверяется непосредственно.

Импликация (1) $\Rightarrow(2)$ вытекает из лемм 3 и 22.

Докажем, что (2) $\Rightarrow(3)$. Без ограничения общности можно считать, что $A=A_{i}$ есть равномерное справа кольцо. Если $A$ есть область, то по лемме 5 область $A$ инвариантна слева. Остается рассмотреть случай, когда равномерное справа кольцо $A$ не является областью. По лемме $15, A$ есть самоинъективное справа локальное кольцо. Остается применить лемму 29.

Лемма доказана.

Лемма 31. Пусть А есть кольц̧о, и пусть все цчиклические правые А-модули вполне цุелозамкнуты.

(1) Если А имеет существвенный нильпотентный левый идеал, то А есть инвариантное кольцо, и А есть конечное прямое произведение ичепных колец.

(2) Если А есть область, то А есть инвариантная слева, равномерная справа, вполне целозамкнутая справа область, и для любого ее ненулевого идеала В факторкольцо A/В есть конечное прямое произведение инвариантных ичепных колец.

Доказательство. Докажем утверждение 1. Так как $A$ имеет существенный нильпотентный левый идеал, мы видим, что кольцо $A$ не имеет прямых сомножителей, являющихся простыми артиновыми кольцами или областями. Поэтому из леммы 30 следует, что $A$ есть конечное прямое произведение инвариантных цепных колец. В частности, $A$ есть инвариантное кольцо.

Докажем утверждение 2. По лемме 30, $A$ есть инвариантная слева, равномерная справа область. Пусть $B$ есть ненулевой собственный идеал в $A$, содержащий ненулевой необратимый элемент $b$. Пусть $h: A \rightarrow A /\left(A b^{2}\right)$ есть естественный кольцевой эпиморфизм. Так как $A$ есть инвариантная слева область и $A b \neq A$, получаем, что $A b^{2}$ является идеалом, $b \notin A b^{2}$, и $h(A b)$ есть ненулевой нильпотентный идеал кольца $h(A)$.

Покажем, что нильпотентный идеал $h(A b)$ кольца $h(A)$ является существенным левым идеалом в $h(A)$. Пусть $a \in A$ и $h(a) \neq h(0)$. Тогда $h(a b) \in h(A a) \cap h(A b)$. Остается доказать, что $h(a b) \neq h(0)$. Допустим противное. Тогда $a b=c b^{2}$ для некоторого $c \in A$. Поэтому $a=c b, h(a)=h(c b)=h(0)$, и получаем противоречие.

Так как $h(A)$ имеет существенный нильпотентный левый идеал, $h(A)$ есть конечное прямое произведение инвариантных цепных колец согласно утверждению 1. Так как 
$A b^{2} \subseteq B$, заключаем, что $A / B$ есть гомоморфный образ кольца $h(A)$. Поэтому $A / B$ есть конечное прямое произведение инвариантных цепных колец.

Лемма доказана.

Лемма 32. Если А есть область, то равносильны следующие условия:

(1) все иุиклические правые А-модули и все цุиклические левые А-модули вполне целозамкнутьл;

(2) А есть вполне цчелозамкнутая инвариантная область, и для любого ее ненулевого идеала $B$ факторкольио $A / B$ есть конечное прямое произведение инвариантных цепных колеи $A_{1}, \ldots, A_{n}$, причем каждое кольцо $A_{i}$ имеет не более двух первичных идеалов, и либо $A_{i}$ есть область, либо для любого нильпотентного непервичного идеала $B$ кольц̧а $A_{i}$ факторкольцฺо $A_{i} / B$ самоинъективно.

Доказательство. Импликация (1) $\Rightarrow(2)$ следует из п. 2 леммы 31 и леммы 29.

Докажем, что $(2) \Rightarrow(1)$. Для этого достаточно доказать, что для любого ненулевого идеала $B$ инвариантной области $A$ кольцо $A / B$ является вполне целозамкнутым правым и левым модулями над $A / B$. Это вытекает из леммы 29 и того, что конечное прямое произведение вполне целозамкнутых колец является вполне целозамкнутым кольцом.

Лемма доказана.

Замечание 1. Теорема 1 вытекает из лемм 30 и 32.

\section{Список литературы}

1. Фейс К., Алгебра: кольца, модули и категории, 2. Мир, Москва, 1979.

2. Brungs H. H., Törner G., Chain rings and prime ideals. Arch. Math. (1976) 27, 253-260.

3. Goel V. K., Jain S. K., $\pi$-injective modules and rings whose cyclics are $\pi$-injective. Commun. Algebra (1978) 6, 59-73.

4. Jeremy L., Modules et anneaux quasi-continus. Canadian Math. Bull. (1974) 17, 217-228.

5. Koehler A., Rings with quasi-injective cyclic modules. Quart. J. Math., Oxford (1974) 25, 51-55.

6. Osofsky B. L., Rings all of whose finitely generated modules are injective. Pacific J. Math. (1964) 14, 645-650.

7. Osofsky B. L., Smith P. F., Cyclic modules whose quotients have all complement submodules direct summands. J. Algebra (1991) 139, 342-354. 